PROCEEDINGS OF THE

AMERICAN MATHEMATICAL SOCIETY

Volume 133, Number 6, Pages 1757-1762

S 0002-9939(04)07732-9

Article electronically published on November 19, 2004

\title{
NON-ZERO BOUNDARIES OF LEIBNIZ HALF-SPACES
}

\author{
FUCHANG GAO
}

(Communicated by N. Tomczak-Jaegermann)

\begin{abstract}
It is proved that for any $d \geq 3$, there exists a norm $\|\cdot\|$ and two points $a, b$ in $\mathbb{R}^{d}$ such that the boundary of the Leibniz half-space $H(a, b)=$ $\left\{x \in \mathbb{R}^{d}:\|x-a\| \leq\|x-b\|\right\}$ has non-zero Lebesgue measure. When $d=2$, it is known that the boundary must have zero Lebesgue measure.
\end{abstract}

\section{INTRODUCTION}

Let $\|\cdot\|$ be an arbitrary norm on $\mathbb{R}^{d}$. For $a, b \in \mathbb{R}^{d}$, the Leibniz half-space $H(a, b)$ is defined as

$$
H(a, b)=\left\{x \in \mathbb{R}^{d}:\|x-a\| \leq\|x-b\|\right\} .
$$

It is natural to study the regularity of $H(a, b)$. In particular, one asks if the Lebesgue measure of the boundary of $H(a, b)$ must be zero. Indeed, such a question arises from the so-called Voronoi partition in information theory and optimal quantization of probability distributions, where one considers a finite intersection of Leibniz half-spaces, called a Voronoi region (cf. [9]). It is a well-known conjecture that the boundary of every Voronoi region has zero Lebesgue measure. (See e.g. [8], Conjecture 1.12.)

Let us also mention that this problem is closely related to metric entropy. Indeed, suppose $S$ is a compact set in $\left(\mathbb{R}^{d},\|\cdot\|\right)$, and suppose $A$ is a finite set in $\mathbb{R}^{d}$ with cardinality $|A|=n$. For each $a \in A$, let

$$
r_{a}=\max \left\{\|y-a\|: y \in \partial \bigcap_{b \in A}[H(a, b) \cap S]\right\},
$$

and define

$$
\varepsilon_{n}:=\inf _{|A|=n} \max _{a \in A} r_{a} .
$$

Then $\varepsilon_{n}$ is just the $n^{\text {th }}$ entropy number of $S$. For a reference on metric entropy, we refer the readers to the excellent book of Carl and Stephani [4].

The problem becomes even more interesting once we notice that the metric entropy number of $S$ is closely related to that of the extreme points of $S$. (See e.g.

Received by the editors August 26, 2003 and, in revised form, February 17, 2004.

2000 Mathematics Subject Classification. Primary 46B20.

Key words and phrases. Leibniz half-space, Voronoi region, metric entropy.

This research was partially supported by NSF grant EPS-0132626 and a seed grant from the University of Idaho.

(C)2004 American Mathematical Society Reverts to public domain 28 years from publication 
[5], 1], 2], 3], 6] and [7].) Such relations and the fact that (2) defines the $n^{\text {th }}$ entropy number of $S$ reflect some regularity of $H(a, b)$.

It can easily be seen that $H(a, b)$ is closed and star-shaped relative to $a$. It can also be proved easily that if $\|\cdot\|$ is strictly convex, then the separator

$$
S(a, b):=\left\{x \in \mathbb{R}^{d}:\|x-a\|=\|x-b\|\right\}
$$

has zero Lebesgue measure, and thus $\partial H(a, b)$, which is a subset of $S(a, b)$, has measure zero. For the $l_{1}^{d}$ or $l_{\infty}^{d}$ norm (which is not strictly convex), $H(a, b)$ is just a finite union of polyhedral sets; thus the boundary has Lebesgue measure zero. Furthermore, when $d=2$, the answer is known to be affirmative. (See e.g. [8], Theorem 1.5.)

In this paper, we will show that in general $H(a, b)$ does not have "very good" regularity. Indeed, for any $d \geq 3$, we will show that the $d$-dimensional Lebesgue measure of $\partial H(a, b)$ may not be zero.

Theorem 1. For any $d \geq 3$, there exists a norm $\|\cdot\|$ on $\mathbb{R}^{d}$, and $a, b \in \mathbb{R}^{d}, a \neq b$, such that $m(\partial H(a, b))=\infty$, where $m$ is the d-dimensional Lebesgue measure, and $H(a, b)$ is the Leibniz half-space defined by (11).

The construction is somewhat technical. In order to help the readers to better understand the idea of the construction and the proof, we first prove that in the case $d=2, m(\partial H(a, b))=0$. As we said earlier, this result has been known, and may already have several proofs. Here we do not intend to reproduce a known proof $([8])$, or produce a somewhat shorter proof; rather, we use this proof for the following two purposes: 1 . to see where the proof breaks down for $d \geq 3$, and give us an idea about where to look for counterexamples; 2 . to make a key observation, which will be essential in the proof of the theorem.

\section{THE CASE $d=2$}

Let $m$ be the Lebesgue measure on $\mathbb{R}^{2}$. We prove that $m(\partial H(a, b))=0$. Because $H(a, b)$ is a closed set, $\partial B=\partial H(a, b)$, where

$$
B=\mathbb{R}^{2} \backslash H(a, b)=\left\{x \in \mathbb{R}^{2}:\|x-b\|<\|x-a\|\right\} .
$$

Clearly, it is enough to prove that any ray starting at $b$ intersects $\partial B$ at most once.

Suppose there is a ray starting at $b$ with direction vector $u$ that intersects $\partial B$ at $x=b+s u$ and $y=b+t u, s<t$. Because $\partial B \subset S(a, b)$, we have $\|x-a\|=\|x-b\|$ and $\|y-a\|=\|y-b\|$. That is, $\|b-a+s u\|=s$ and $\|b-a+t u\|=t$. This implies that for any $p, q>0$,

$$
\|p(b-a+s u)+q(t-s) u\|=\|p(b-a+s u)\|+\|q(t-s) u\| .
$$

Indeed, by linearity, it is enough to prove the case when $p=1, q \leq 1$ (or $q=1$, $0<p \leq 1)$. Suppose $p=1,0<q \leq 1$. Then

$$
\begin{aligned}
t & =\|b-a+t u\| \\
& =\|b-a+s u+(t-s) u\| \\
& \leq\|b-a+s u+q(t-s) u\|+(1-q)\|(t-s) u\| \\
& \leq\|b-a+s u\|+q\|(t-s) u\|+(1-q)\|(t-s) u\| \\
& =s+q(t-s)+(1-q)(t-s) \\
& =t .
\end{aligned}
$$


The two ends are equal, so

$$
\|b-a+s u+q(t-s) u\|=\|b-a+s u\|+q\|(t-s) u\|,
$$

and (4) is thus valid.

Now, let $w=p(b-a)+b+\lambda u$ where $p \geq 0$ and $\lambda \geq(p+1) s$. By (4),

$$
\begin{aligned}
\|w-a\| & =\|(p+1)(b-a)+\lambda u\| \\
& =\|(p+1)(b-a+s u)+(\lambda-(p+1) s) u\| \\
& =(p+1)\|b-a+s u\|+\|(\lambda-(p+1) s) u\| \\
& =(p+1) s+(\lambda-(p+1) s) \\
& =\lambda
\end{aligned}
$$

and

$$
\begin{aligned}
\|w-b\| & =\|p(b-a+s u)+(\lambda-p s) u\| \\
& =\|p(b-a+s u)\|+\|(\lambda-p s) u\| \\
& =p s+(\lambda-p s) \\
& =\lambda .
\end{aligned}
$$

Thus, for any fixed $\lambda \geq s$, each point $w$ on the line segment

$$
\left\{w=p(b-a)+b+\lambda u: 0 \leq p \leq \frac{\lambda}{s}-1\right\}
$$

satisfies $\|w-a\|=\|w-b\|=\lambda$. In particular, the entire planar section

$$
V:=\left\{w=p(b-a)+b+\lambda u: \lambda \geq s, 0 \leq p \leq \frac{\lambda}{s}-1\right\}
$$

is contained in $S(a, b) \subset H(a, b)$.

Because $H(a, b)$ is star-shaped, the extended open section

$$
V^{+}:=\left\{v=q(b-a)+b+\lambda u: \lambda>s,-1<q<\frac{\lambda}{s}-1\right\}
$$

is contained in $H(a, b)$. Indeed, for any $v=q(b-a)+b+\lambda \in V^{+}$with $-1<q<0$, $\lambda>s$, let $w=b+\frac{\lambda}{1+q} \cdot u$. Because $v$ lies on the line segment between $a$ and $w$, we have $\|w-a\|=\|w-v\|+\|v-a\|$. On the other hand, because $w \in V$, we have $\|w-a\|=\|w-b\|$. Thus,

$$
\|v-b\| \geq\|w-b\|-\|w-v\|=\|w-a\|-\|w-v\|=\|v-a\| .
$$

So, $v \in H(a, b)$. This proves that $V^{+} \subset H(a, b)$.

Clearly $y=b+t u \in V^{+}$. When $d=2, y=b+t u$ is an interior point of $V^{+}$, so an interior point of $H(a, b)$, contradicting the assumption that $y \in \partial B$. This implies that in $\mathbb{R}^{2}$, any ray starting at $b$ intersects $\partial B$ at most once. This implies $m(\partial H(a, b))=m(\partial B)=0$.

\section{THE CASE $d=3$}

The proof in the previous section tells us the following fact, which will be used later in the proof. 
Fact: If $b+s u$ and $b+t u$ are both in the separator $S(a, b)$, then for $\lambda>s$, and any point $w$ on the line segment

$$
\left\{w=p(b-a)+b+\lambda u: 0 \leq p \leq \frac{\lambda}{s}-1\right\},
$$

$\|w-a\|=\|w-b\|=\lambda$.

We also notice from the proof above that for any ray starting at $b$, either

(1) it intersects the separator $S(a, b)$ at most once; or

(2) there exists a planar section on the plane generated by this ray and the point $a$, such that the entire planar section is contained in the separator.

In $\mathbb{R}^{2}$, the first case prohibits the second case to happen in a large neighborhood. This leads to the regularity of the separator; while in $\mathbb{R}^{d}, d>2$, both cases can coexist on a very close neighborhood. This leads to the irregularity of the separator.

Our idea is then to construct a Banach space norm in $\mathbb{R}^{d}, d \geq 3$, such that there are "many" directions in which the ray starting at $b$ intersects the separator at most once; while in other directions in the close neighborhood, there exist planar sections contained in the separator. When this is done, we will show that for each of those planar sections, half of the section is contained in the boundary of $H(a, b)$. Thus, by assigning "sufficiently many" directions for each case, we can make the boundary of $H(a, b)$ have large Lebesgue measure.

3.1. Construction. We start with the closed interval $[-1,1]$, and construct a Cantor-like set. First, remove an open interval of length $10^{-1}$ from the middle; that is, remove $(-1 / 20,1 / 20)$. We obtain two disjoint intervals. Next, from each of the two intervals, we remove an open interval of length $10^{-2}$ in the middle. We then obtain four disjoint closed intervals. Then, from each of the intervals, we remove an open interval of length $10^{-3}$ in the middle. Continue this procedure. The remaining set is a closed subset of $[-1,1]$ with Lebesgue measure $15 / 8$. We denote this set by $E$, and denote $[-1,1] \backslash E$ by $G$. Clearly, $E=\partial(G)$.

Next, we define $F=\left\{(\sin (\pi t / 2), \cos (\pi t / 2)) \in \mathbb{R}^{2}: t \in E\right\}$. $F$ is a closed subset of the unit circle $T$.

Then, we define a set $D$ as the closed convex hull of $F \cup(-F)$. $D$ is a symmetric convex closed set in $\mathbb{R}^{2}$.

Now, we define a symmetric convex body $K \subset \mathbb{R}^{3}$ as the closed convex hull of $\{-1\} \times D,\{0\} \times T$ and $\{1\} \times D$. The symmetry of $K$ follows from the symmetry of $F$. $K$ can be imagined as a short log with some wood pared off symmetrically at both ends.

Finally, we define a norm $\|\cdot\|_{K}$ in $\mathbb{R}^{3}$ by

$$
\|x\|_{K}=\inf \{t \in \mathbb{R}: t x \in K\} .
$$

3.2. Proof. We will show that under the norm $\|\cdot\|_{K}$ defined above, if we choose $a=(-1,0,0)$ and $b=(1,0,0)$, and let $m$ be the three-dimensional Lebesgue measure, then $m(\partial H(a, b))=\infty$.

Claim 1. For each $(y, z) \in F \cup(-F), V(y, z) \subset S(a, b)$, where $V(y, z)$ is a planar section defined by

$$
V(y, z):=\{(x, t y, t z): t \geq 1,-(t-1) \leq x \leq t-1\} .
$$


Proof. Let $(x, t y, t z) \in V(y, z)$. Because $(-1, y, z) \in\{-1\} \times D \subset K,(1, y, z) \in$ $\{1\} \times D \subset K$, and $-1<\frac{x+1}{t} \leq 1$, we have $\left(\frac{x+1}{t}, y, z\right) \in K$ by the convexity of $K$. So,

$$
\|(x, t y, t z)-a\|_{K}=\|(x+1, t y, t z)\|_{K}=t\left\|\left(\frac{x+1}{t}, y, z\right)\right\|_{K} \leq t .
$$

On the other hand, for any $\lambda>1, \lambda \cdot\left(\frac{x+1}{t}, y, z\right) \notin K$. Therefore $\left\|\left(\frac{x+1}{t}, y, z\right)\right\|_{K}=1$. Hence $\|(x, t y, t z)-a\|_{K}=t$.

Similarly, we can prove that $\|(x, t y, t z)-b\|_{K}=t$. Thus, $(x, t y, t z) \in S(a, b)$. Hence $V(y, z) \subset S(a, b) \subset H(a, b)$.

Claim 2. For each $(y, z) \in T \backslash[F \cup(-F)], L(y, z) \cap H(a, b)=\emptyset$, where $L(y, z)$ is an open half-plane defined by

$$
L(y, z):=\left\{(x, t y, t z) \in \mathbb{R}^{3}: x>0, t \in \mathbb{R}\right\} .
$$

Proof. By the symmetry of $K$, and the fact that $a=-b, S(a, b)$ contains the $y z$ plane. Suppose $S(a, b)$ also contains $(x, t y, t z) \in L(y, z)$. Then the ray from $a$ to $(x, t y, t z)$ intersects $S(a, b)$ in at least two points, one at $(x, t y, t z)$, the other on the $y z$-plane. Thus, by what we have concluded in the beginning of this section, for $\lambda>$ 1 large enough, any point $w$ on the line segment $\{(p, \lambda y, \lambda z):-1 \leq p \leq 0\}$ satisfies $\|w-a\|_{K}=\lambda$. In particular, $\|(-1, \lambda y, \lambda z)-a\|_{K}=\|(0, \lambda y, \lambda z)-a\|_{K}$. Thus, $\|(1, \lambda y, \lambda z)\|_{K}=\|(0, \lambda y, \lambda z)\|_{K}=\lambda$, i.e. $\|(1 / \lambda, y, z)\|_{K}=1$. Thus, $(1 / \lambda, y, z) \in K$. Because this point lies in the convex hull of $\{0\} \times T$ and $\{1\} \times(F \cup(-F))$, we have

$$
(1 / \lambda, y, z)=\frac{1}{\lambda} \cdot(1, \alpha, \beta)+\left(1-\frac{1}{\lambda}\right) \cdot(0, \gamma, \delta),
$$

where $(1, \alpha, \beta) \in\{1\} \times(F \cup(-F))$ and $(0, \gamma, \delta) \in\{0\} \times T$. This implies that

$$
\left[\frac{\alpha}{\lambda}+\left(1-\frac{1}{\lambda}\right) \gamma\right]^{2}+\left[\frac{\beta}{\lambda}+\left(1-\frac{1}{\lambda}\right) \delta\right]^{2}=y^{2}+z^{2}=1
$$

This is impossible unless $(\alpha, \beta)=(\gamma, \delta)= \pm(y, z)$. This contradicts the assumptions that $(y, z) \in T \backslash[F \cup(-F)]$, and $(\gamma, \delta) \in F \cup(-F)$.

So far, we have proved that $L(y, z) \cap S(a, b)=\emptyset$.

To finish the proof of the claim, we need to show $(H(a, b) \backslash S(a, b)) \cap L(y, z)=\emptyset$. Suppose not; then there exists $w \in L(y, z)$ such that $\|w-a\|_{K}<\|w-b\|$. By the continuity of the norm, at some point $v$ on the line segment between $w$ and $b$, we have $\|v-a\|_{K}=\|v-b\|_{K}$. That is, $v \in S(a, b)$. Because $b \in L(y, z)$, and $L(y, z)$ is convex, we have $v \in L(y, z)$, contradicting the fact that $L(y, z) \cap S(a, b)=\emptyset$.

Thus, $L(y, z) \cap H(a, b)=\emptyset$, proving the claim.

Now, we will finish the proof of Theorem 1 in the case $d=3$. Note that $F \cup(-F)$ are the limit points of $T \backslash[F \cup(-F)]$. Thus, Claims 1 and 2 imply that for each $(y, z) \in F \cup(-F)$, the planar section

$$
V^{+}(y, z):=\{(x, t y, t z): t \geq 1,0 \leq x \leq t-1\}
$$

is contained in $\partial H(a, b)$.

Let $\sigma$ be the Haar measure on $T$. It is clear by the construction of $F$ that $\sigma(F \cup(-F))=15 / 16$. Therefore $\bigcup_{(y, z) \in F \cup(-F)} V^{+}(y, z)$ has infinite Lebesgue measure. Hence, $m(\partial H(a, b))=\infty$. 


\section{THE CASE $d>3$ AND REMARKS}

The case $d>3$ can be proved similarly. However, an easier way is to use $K \times[-1,1]^{d-3}$ as the symmetric convex body in the definition of $\|\cdot\|$.

For the readers who are interested in knowing the exact boundary of the Leibniz half-space given in the last section, we have

$\partial H(a, b)=\left(\{0\} \times \mathbb{R}^{2}\right) \cup\{(x, r \cos (t \pi / 2), \pm r \sin (t \pi / 2)): r \geq 1,0 \leq x \leq r-1, t \in E\}$ and $\partial H(b, a)=-\partial H(a, b)$.

The results of this paper indicate the irregularity of $H(a, b)$ in general. On the other hand, $H(a, b)$ does have some regularity. For example, the proof given in the paper implies that in general either $m(\partial H(a, b))=0$ or $m(\partial H(a, b))=\infty$.

\section{REFERENCES}

1. Keith Ball and Alain Pajor, The entropy of convex bodies with "few" extreme points, Geometry of Banach spaces (Strobl, 1989), London Math. Soc. Lecture Note Ser., vol. 158, Cambridge Univ. Press, Cambridge, 1990, pp. 25-32. MR93b:46024

2. Bernd Carl, Entropy numbers of diagonal operators with an application to eigenvalue problems, J. Approx. Theory 32 (1981), no. 2, 135-150. MR83a:47024

3. Bernd Carl, Ioanna Kyrezi, and Alain Pajor, Metric entropy of convex hulls in Banach spaces, J. London Math. Soc. (2) 60 (1999), no. 3, 871-896. MR.2001c:46019

4. Bernd Carl and Irmtraud Stephani, Entropy, compactness and the approximation of operators, Cambridge Tracts in Mathematics, vol. 98, Cambridge University Press, Cambridge, 1990. MR 92e:47002

5. R. M. Dudley, Universal Donsker classes and metric entropy, Ann. Probab. 15 (1987), no. 4, 1306-1326. MR88g:60081

6. Fuchang Gao, Metric entropy of convex hulls, Israel J. Math. 123 (2001), 359-364. MR2002c:46044

7. (2004), no. 4, 460-468.

8. Siegfried Graf and Harald Luschgy, Foundations of quantization for probability distributions, Lecture Notes in Mathematics, vol. 1730, Springer-Verlag, Berlin, 2000. MR2001m:60043

9. Atsuyuki Okabe, Barry Boots, and Kōkichi Sugihara, Spatial tessellations: concepts and applications of Voronő diagrams, Wiley Series in Probability and Mathematical Statistics: Applied Probability and Statistics, John Wiley \& Sons Ltd., Chichester, 1992, With a foreword by D. G. Kendall. MR94a:52033

Department of Mathematics, University of Idaho, Moscow, Idaho 83843 